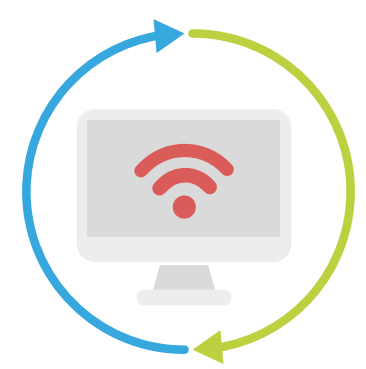

\title{
A TELERREGULAÇÃO NA AMPLIAÇÃO DO ACESSO AOS SERVIÇOS DE SAÚDE DO MUNICÍPIO DE NATAL
}

THE TELERREGULATION INCREASING THE ACCESS TO HEALTH SERVICES IN NATAL CITY

\section{Karla Mônica Dantas Coutinho}

Mestranda em Gestão e Inovação em Saúde pela UFRN. Especialista em Informática em Saúde e Gestão de Negócios e Qualidades de Alimentos. Graduada em Nutrição pela UFRN. Membro do Núcleo Técnico-científico de Telessaúde do RN. Pesquisadora do Laboratório de Inovação Tecnológica em Saúde

$$
\text { (LAIS - HUOL - UFRN). }
$$

E-mail: kmdcoutinho@gmail.com

Antônio Higor Freire de Morais

Doutor em Engenharia Elétrica e de Computação pela UFRN. Mestre em Engenharia Elétrica pela UFRN. Professor de Sistemas de Informação do IFRN. Membro do Núcleo Avançado de Inovação Tecnológica (NAVI/IFRN). Pesquisador do Laboratório de Inovação Tecnológica em Saúde (LAIS/HUOL/UFRN).

Coordenador estratégico do Núcleo Técnico-científico de Telessaúde do RN.

E-mail: higormorais@gmail.com

Edson da Silva Freitas

Mestrando em Ensino na Saúde pela UFRN. Graduado em Medicina pelo Centro Universitário Lusíada. Atua na Residência Médica em Medicina de Família e Comunidade. Telerregulador do Núcleo Técnico-científico de Telessaúde do RN. Professor da UFRN. Preceptor da Residência de Medicina de Família e Comunidade da UFRN e tutor de prática da Universidade Potiguar.

E-mail: edson.mfc@gmail.com

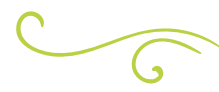

\section{RESUMO}

O Programa Nacional Telessaúde Brasil Redes é uma iniciativa instituída em âmbito nacional pelo Ministério da Saúde, desde 2007, e busca melhorar a qualidade do atendimento na atenção primária do Sistema Único de Saúde (SUS), atuando como retaguarda assistencial e educacional, por meio de ferramentas de tecnologias da informação e comunicação (TIC), garantindo a promoção da educação permanente aos profissionais da saúde. $\bigcirc$ presente artigo refere-se a um estudo de caso com a finalidade de proporcionar critérios para a solicitação do exame de colonoscopia pela rede de saúde do município de Natal/ $\mathrm{RN}$, que contava com uma fila de espera de mais de 4.000 solicitações de colonoscopias. Os resultados alcançados por meio da plataforma de telessaúde do Rio Grande do Norte como ferramenta de transformação do processo de regulação das solicitações de exames de colonoscopia evitaram o encaminhamento desnecessário de mais de 30\% das solicitações de exames. No período de novembro de 2017 a agosto de 2018, foram 
realizadas 608 solicitações de exames de colonoscopia. Todas as solicitações foram atendidas no prazo. A ferramenta de regulação de exames se configura como uma importante estratégia para avaliação dos pedidos de exames, bem como para qualificar tais pedidos e contribuir para a formação continuada dos profissionais de saúde.

Palavras-chave: Atenção Primária em Saúde. Telessaúde. Telerregulação. Colonoscopia e Sistema Único de Saúde.

\section{ABSTRACT}

The National Program Telehealth Brazil Networks is an initiative instituted at the national level by the Ministry of Health, since 2007 , search to improve the quality of care in the primary attention of the Unified Health System (SUS), acting as an aid and educational rearguard, through tools of information and communication technologies (ICT), ensuring the promotion of permanent education for health professionals. The present article refers to a case study with the purpose of providing criteria for the request of the colonoscopy exam by the health network of the city of Natal / RN, which had a queue of more than 4,000 requests for colonoscopies. The results achieved by the telehealth platform of Rio Grande do Norte as a tool for transforming the process of regulation of requests for colonoscopy exams avoided the unnecessary routing of more than $30 \%$ of the exam requests. In the period from november/17 to august/18, 608 requests were made for colonoscopy exams. All requests were met on time. The examination regulation tool is an important strategy for the evaluation of the examination requests, as well as to qualify such applications and contribute to the continuing training of health professionals.
Keywords: Primary Health Care. Telehealth. Telerregulation. Colonoscopy and Single Health System.

\section{INTRODUÇÃO}

O Sistema Único de Saúde (SUS) surgiu com o intuito de prestar uma nova forma de assistência, na qual a saúde passa a ser direito de todos que o Estado deve oportunizar. Sendo assim, o sistema segue princípios e diretrizes que visam uma assistência universal, integrada e igualitária, cujo acesso se dá pela Atenção Primária à Saúde (APS), estruturada pela Estratégia de Saúde da Família (ESF) (NETO et al., 2018).

A Atenção Primária à Saúde foi descrita inicialmente num contexto de Redes de Atenção à Saúde (RAS) e conceituada, contemporaneamente, como o nível preferencial de acesso para a atenção à saúde, responsável por um atendimento integral, longitudinal e coordenado. Seu papel de ordenadora e de centro de comunicação das RAS, definido no marco legal brasileiro, não tem sido atingido. Sua materialização no país apresenta ainda grandes heterogeneidades em termos de acesso, qualidade e custo da atenção à saúde. A fonte dessa heterogeneidade tem como causas, entre outras, deficiências na formação profissional, déficits estruturais e baixa incorporação tecnológica (SCHMITZ; HARZHEIM, 2017).

O Telessaúde Brasil Redes na Atenção Primária à Saúde, componente do Programa de Requalificação das Unidades Básicas de Saúde (UBS), instituído pela Portaria $n^{\circ}$ 2.554, de 28 de outubro de 2011, objetiva potencializar a qualificação da Atenção Primária/Estratégia de Saúde da Família, ao estimular o uso das Tecnologias de Informação e Comunicação (TIC) para atividades a distância relacionadas à saúde, podendo possibilitar a interação entre profissionais de saúde, bem como o acesso remoto a recursos de apoio educacional, clínico e diagnóstico (BRASIL, 2015). 
A partir dessa portaria os municípios puderam apresentar projetos ao Departamento de Atenção Básica (DAB) da Secretaria de Atenção à Saúde (SAS), com repasse de recurso fundo a fundo, assim como também surgiram novos desenhos e formatos de núcleos de telessaúde (FIGUEIREDO; GUEDES, 2018).

Os núcleos de telessaúde são formados por instituições formadoras e de gestão e/ou serviços de saúde responsáveis pela formulação, desenvolvendo atividades técnicas, científicas e administrativas para planejar, executar, monitorar e avaliar as ações do telessaúde. Os núcleos de telessaúde podem ter abrangência estadual, interestadual ou intermunicipal, (COUTINHO et al., 2017), por meio de trabalho compartilhado das secretarias estaduais e municipais de saúde, instituições de ensino e serviços de saúde, oferecendo apoio permanente aos profissionais em diferentes serviços e distintos locais (NILSON et al., 2018). Já os pontos de telessaúde são serviços de saúde a partir dos quais os profissionais da área da saúde do SUS demandam/utilizam as ações de telessaúde (SILVA, 2017).

A regulação da assistência à saúde tem a função primordial de ordenar o acesso às ações e aos serviços de saúde, em especial, à alocação prioritária de consultas médicas e aos procedimentos diagnósticos e terapêuticos para pacientes com maior risco, necessidade e/ou indicação clínica oriundos dos diversos serviços de saúde em tempo oportuno. Complementarmente, a regulação deve servir de filtro aos encaminhamentos desnecessários, devendo selecionar o acesso dos pacientes às consultas e/ou aos procedimentos apenas quando eles apresentarem indicação clínica para realizá-los, evitando a exposição dos pacientes a consultas e/ou a procedimentos desnecessários. Além disso, otimiza o uso dos recursos em saúde, impede deslocamentos desnecessários e traz maior eficiência e equidade à gestão das listas de espera (BRASIL, 2016).
Existem experiências exitosas de pactuações de regulação entre telessaúde e secretarias municipais de saúde em vários locais do país, como o exemplo do estado de Santa Catarina, que levantou dados de encaminhamentos médicos após regulação do telessaúde. $\bigcirc$ estudo percebeu uma queda expressiva no número de casos encaminhados via SISREG após o início do fluxo que inclui a teleconsultoria na discussão de casos previamente aos encaminhamentos (CORTESE et al., 2017).

O Programa Telessaúde vem ganhando visibilidade e se tornando foco de interesse da gestão a partir das evidências apresentadas, tornando-se uma ferramenta importante de regulação, atuando na redução de encaminhamentos para especialidades, reduzindo filas de espera por atendimento com especialistas e custos para a gestão (FIGUEIREDO et al., 2015).

Sabe-se que a integração dos sistemas de informação no setor público definiria o sucesso no alcance dos objetivos e possibilitaria a redução de custos de desenvolvimento. Se setores afins focassem em suas áreas específicas de atendimento e, também, preparassem seus bancos de dados para a troca de informação, haveria um consequente aproveitamento de dados, e isso reduziria, significativamente, o retrabalho. Em âmbito nacional, há uma tendência na busca de alternativas viáveis de reaproveitamento das informações públicas e de que o foco deva ser a integração dos dados produzidos (GEREMIAS; JACOBSEN; PEREIRA, 2013).

A colonoscopia é um exame de imagem que permite a visualização do interior de todo o cólon. $O$ instrumento utilizado é um tubo flexível com cerca de um metro de comprimento e um centímetro de diâmetro. $\mathrm{Na}$ extremidade final desse tubo existe uma minicâmera, que transmite para um monitor colorido, as imagens do interior do cólon e do íleo terminal. Essas imagens são fotografadas e impressas (SEGUNDA OPINIÃO FORMATIVA, 2013). É um exame 
de custo relativamente alto e, por isso, sua oferta deve ser restrita às reais indicações de realização.

A solicitação inadequada de exames, muitas vezes, gera gargalos no sistema de saúde, no qual a demanda supera a oferta, criando filas de espera que comprometem a saúde dos indivíduos. Esses gargalos requerem, para a sua superação, que se constituam estratégias que impactem na Atenção Primária à Saúde (APS) nos processos de regulação do acesso (desde os serviços solicitantes até as centrais de regulação), bem como na organização da atenção especializada (BRASIL, 2016).

Nessa perspectiva o estudo tem como objetivo proporcionar melhores critérios de regulação para a solicitação de exames de colonoscopia na rede de saúde de Natal, fazendo uso da plataforma do Núcleo de Telessaúde do Rio Grande do Norte, otimizando o processo e melhorando a resolutividade dos agendamentos.

\section{METODOLOGIA}

Trata-se de um estudo de caso de abordagem qualitativa com a finalidade de proporcionar critérios para a solicitação do exame de colonoscopia e discernir melhor as prioridades agilizando o processo para o paciente mais necessitado. O Núcleo de Telessaúde do RN implementou a regulação de exames de colonoscopia na rede de saúde de Natal, em parceria com o Departamento de Atenção Básica (DAB), Departamento de Atenção Especializada (DAE) e Departamento de Regulação da Secretaria Municipal de Saúde (SMS).

O município de Natal, em outubro de 2017, na época do lançamento do projeto-piloto, contava com uma fila de espera de mais de 4.000 solicitações de colonoscopias. A partir da adoção do sistema do telessaúde para telerregulação das solicitações de colonoscopias, a SMS definiu que todos os pedidos com mais de 6 meses de espera no Sistema de Regulação (SISREG) fossem refeitos com o intuito de reavaliar suas indicações (PREFEITURA MUNICIPAL DO NATAL, 2018).

O fluxo de marcações é iniciado pelo médico solicitante, que insere a solicitação do exame no sistema de telessaúde acompanhando todo o processo até o agendamento deste. Em seguida, a solicitação será avaliada pelo Regulador, membro do telessaúde, que é um profissional médico. Uma vez aprovada, será acessada pela Central de Regulação, da Secretaria Municipal de Saúde (SMS), que então marca o exame no Sistema de Centrais de Regulações (SISREG), de acordo com a disponibilidade de vagas. O Administrativo UBS (Unidades Básicas de Saúde) é o profissional que acompanha o processo, visualizando os exames que são regulados e agendados, podendo entrar em contato com o usuário. A Figura 1 ilustra o fluxo do processo. 


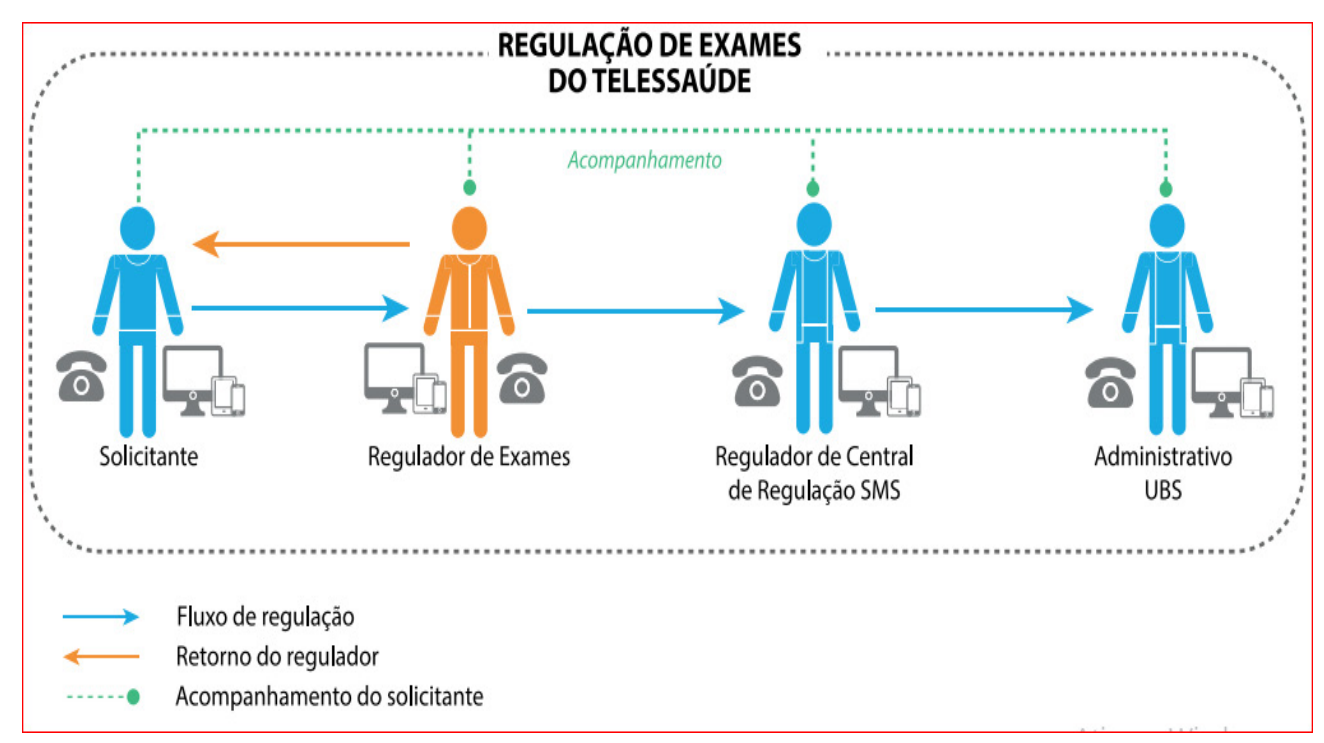

Figura 1 - Regulação de Exames Telessaúde. Fonte: Autoria própria (2018).

Cada um desses profissionais é capacitado para poder utilizar o sistema de regulação de exames do telessaúde, cuja capacitação dura cerca de 40 minutos para apresentação do processo. Esse sistema pode ser acessado pela URL https://teleconsultoria.telessaude. ufrn.br, podendo ser feito via computador ou smartphone com acesso à internet. A capacitação tem o intuito de prevenir equívocos na solicitação e evitar absenteísmo dos pacientes após agendamento.

Na plataforma do telessaúde, o médico insere os principais dados de identificação do paciente, assim como o resumo clínico, exames realizados, tratamentos prévios, impressão diagnóstica e seu respectivo CID (Classificação Internacional de Doenças) ou CIAP (Classificação Internacional da Atenção Primária). Além disso, o exame pode ter caráter eletivo ou urgente. Uma vez que o médico solicitante tenha feito o pedido de exame, o telessaúde tem $72 \mathrm{~h}$ corridas para dar uma resposta.

O regulador de exames do telessaúde avalia a solicitação, podendo regular ou não. Caso seja regulado, o caráter da solicitação pode ser alterado para eletivo ou urgente a partir dos dados clínicos apresentados. Caso não seja regulado, o regulador do telessaúde pode reivindicar ao médico solicitante maior detalhamento e/ou orientar sobre outros procedimentos. Nessa situação, a devolução é acompanhada de justificativa que pode incluir a solicitação de mais dados para autorização do exame ou de material de educação permanente, indicando outras condutas possíveis para diagnóstico e manejo do paciente.

\section{RESULTADOS E DISCUSSÃO}

O Núcleo de Telessaúde RN realizou, no período de outubro de 2017 a julho de 2018, 10 capacitações presenciais atingindo um público de 113 profissionais solicitantes médicos das UBS e das unidades especializadas, reguladores e administradores das UBS do município de Natal, para o uso da plataforma do telessaúde na regulação de exames. Essas ações de capacitação dos profissionais foram fundamentais para uma forte adesão ao sistema de telerregulação do Telessaúde RN e para que o fluxo de implantação pudesse ocorrer de forma mais fluida.

No período de novembro de 2017 até agosto de 2018, foram geradas 608 solicitações de exames de colonoscopia na plataforma do telessaúde, com 192 devoluções (31,5\%), uma fila de espera de 187 pedidos e 229 colonoscopias agendadas 
e realizadas pela SMS, conforme ilustrado no Gráfico 1. Destas, 100 de caráter urgente foram agendadas pela regulação do município no prazo máximo de uma semana. No que compete ao Núcleo interestadual de Telessaúde RN/PB, todas as solicitações de exames foram atendidas no prazo máximo para regulação - 72 horas, o qual é preconizado pela nota técnica número 50/2015 DEGES/SGTES/MS e DAB/SAS/MS do Ministério da Saúde, que estabelece o funcionamento dos serviços do sistema de telessaúde no Brasil (BRASIL, 2015).

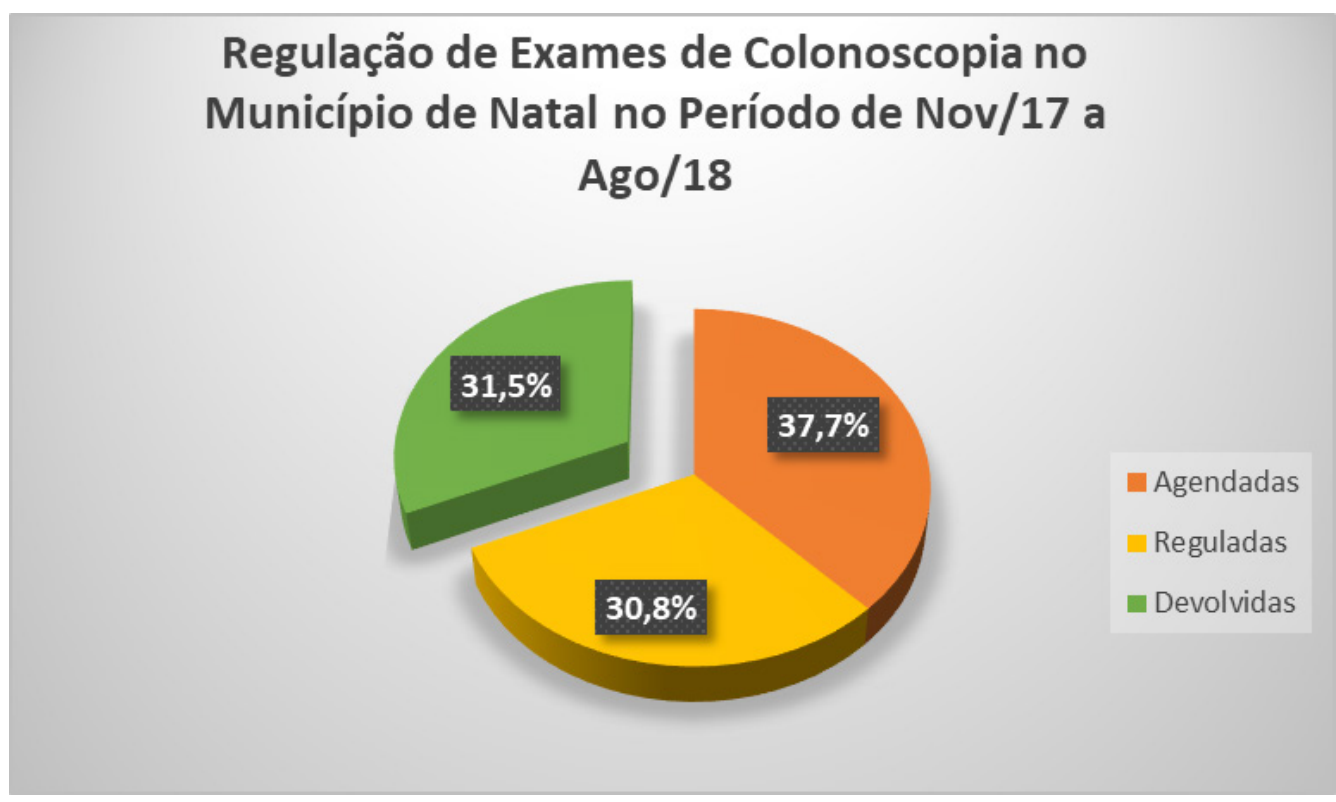

Gráfico 1 - Regulação de Exames de Colonoscopia no Município de Natal.

Fonte: Autoria própria (2018).

A partir dessa primeira análise, é possível verificar que o fluxo de telerregulação com o apoio da telessaúde evitou o encaminhamento desnecessário de mais de $30 \%$ das solicitações de exames feitas no período. Ou seja, 192 possíveis solicitações que iriam para a fila de regulação foram solucionadas com o auxílio do telessaúde, a partir do qual foram feitas sugestões de manejo na Atenção Básica. Nesse aspecto, há um ganho direto tanto para o usuário do serviço, que recebe uma atenção e um cuidado de forma mais célere, quanto para o sistema de saúde, que pode direcionar os seus recursos de forma mais eficiente.

Somente a partir de uma análise detalhada por parte de um profissional devidamente capacitado, é possível verificar a qualidade das solicitações de exames que estão sendo feitas. Desse modo, com a identificação de solicitações de exames incompletas ou fora dos protocolos de indicação definidos pelo Ministério da Saúde, permitiu-se, por meio da telerregulação, criar uma ferramenta para diagnóstico e educação permanente dos profissionais de saúde. Isso porque a recusa dos pedidos de solicitação de exames é centrada na observação de uma necessidade concreta de aprendizado do médico da Atenção Básica, com potencial de aumentar sua resolutividade nos casos de surgimento de demandas futuras que sejam semelhantes às já ocorridas. Além disso, observa-se um importante impacto positivo na maior agilidade para realização dos exames de colonoscopia, principalmente os de caráter urgente, promovendo assim uma maior equidade.

Por outro lado, o sistema também contribui sobremaneira para que a gestão 
municipal possa avaliar as demandas de pedidos de exames e redirecionar as políticas de saúde com base nessas informações. Assim, o sistema de telessaúde para regulação de exames de colonoscopia se consolida como ferramenta que apoia, por um lado, o profissional de saúde que precisa de algum suporte de especialista, por outro, o usuário final que acaba por receber um serviço de saúde mais eficiente.

Além disso, a própria gestão dos recursos pode se dar de forma mais assertiva, posto que com base na análise das demandas, com uma regulação mais qualificada, o gestor pode de fato avaliar a real escala e necessidade a ser atendida. A partir de uma análise mais detalhada também é possível verificar casos de concentrações de ocorrências em determinada região ou bairro do município e, por conseguinte, a definição de estratégias para identificar e solucionar a causa.

Um dos principais entraves encontrados na implementação do processo de regulação das colonoscopias por meio do uso da ferramenta de teleconsultoria desenvolvida pelo Núcleo de Telessaúde do RN reside no fato de que o SISREG não permite, atualmente, a integração de outros sistemas. Devido a esse impedimento, faz-se necessário o estabelecimento de um estágio manual em que os dados das solicitações de exames reguladas pelo telessaúde sejam inseridos no SISREG manualmente. Dessa forma, acaba por ser gerado um retrabalho, pois os dados da solicitação já se encontravam cadastrados na plataforma do telessaúde.

Esse fato é de extrema relevância, inclusive para uma boa aceitação por parte dos profissionais da regulação do município na utilização da ferramenta do telessaúde. Nesse sentido, o Núcleo de Telessaúde do RN já vem estabelecendo alguns diálogos com o Ministério da Saúde e o DATASUS para que seja possibilitada essa integração entre as ferramentas. É importante destacar que outros núcleos de telessaúde também têm o interesse para que essa abertura seja implementada.

\section{CONSIDERAÇÕES FINAIS}

processo de interação entre as ações promovidas pelo telessaúde e a Secretaria Municipal de Saúde de Natal demonstram um claro aumento da eficiência de prestação dos serviços de saúde com contrapartida direta para qualificação dos profissionais médicos da Atenção Básica. Nesse contexto, o processo de regulação é um dos principais pilares para que os serviços de saúde sejam corretamente direcionados e os recursos possam ser acionados conforme as necessidades.

Além disso, a regulação, quando realizada tendo como base diretriz firmada em processo sólido, proporciona que as ofertas de serviços de saúde sejam corretamente guiadas. As filas de espera tendem a diminuir, uma vez que solicitações desnecessárias ocasionadas por pedidos mal formulados não entram na fila; com isso, aquele usuário que apresenta uma real necessidade pelo uso do serviço não deverá ficar desassistido.

Foi possível observar, a partir dos resultados obtidos, que o delineamento de 72 horas para atendimento ao pedido do médico solicitante torna o tempo de resposta à solicitação muito menor do que era praticado anteriormente (havia algumas solicitações de exames com mais de 6 meses de espera). Dessa forma, fica evidente, mais uma vez, a importância das pesquisas realizadas por instituições de ensino atuando como provedor de tecnologia e inovação para a sociedade. 


\section{REFERÊNCIAS}

BRASIL. Ministério da Saúde. Custeio dos Núcleos de Telessaúde.

Manual Instrutivo. Brasília: Ministério da Saúde, 2015.

BRASIL. Ministério da Saúde. Universidade Federal do Rio Grande do Sul. Proctologia. Brasília: Ministério da Saúde, 2016. (Protocolos de encaminhamento da atenção básica para a atenção especializada, v. 7). Disponível em: https://www.ufrgs.br/ telessauders/documentos/protocolos_resumos/protocolo_ms_ proctologia_janeiro_2016.pdf. Acesso em: 4 ago. 2018.

BRASIL. Ministério da Saúde. Nota Técnica n 50/2015-DEGES/ SGTES/MS de 15 de outubro de 2015. Diretrizes para ofertas de atividades do Programa Nacional Telessaúde Brasil Redes. Disponível em: http://smart.telessaude.ufrn.br/static/smart/Nota\%20 Técnica\%20n\%2050_DEGES_SGTES_MS_\%20e\%20DAB_SAS_ MS\%20Diretrizes\%200fertas\%20das\%20atividadesTelessaúde\%20 Brasil\%20Redes.pdf. Acesso em: 23 set. 2018.

CORTESE, M. et al. Avanço da Teleconsultoria no Estado de Santa Catarina: uma parceria da Regulação do Estado com o Núcleo Telessaúde. Revista Catarinense de Saúde da Família, ano VII, n. 14, out. 2017.

COUTINHO, K. M. D. et al. Capacitação dos Profissionais de Saúde em Ferramentas de Telessaúde: uma abordagem do núcleo de telessaúde do rio grande do Norte. Revista Brasileira de Inovação Tecnológica em Saúde, v. 7, n. 1, 2017.

FIGUEIREDO, A. M.; GUEDES, T. A. L. Programa Nacional Telessaúde Brasil Redes. In: SEDIS/UFRN (ed.). A Telessaúde no Brasil e a Inovação Tecnológica na Atenção Primária. 2. ed. Natal: [s.n.], 2018. p. 27-46.

FIGUEIREDO, A. M. de et al. Curso autoinstrucional em telessaúde: uma visão geral. Revista Brasileira de Inovação Tecnológica em Saúde, v. 4, p. 43-50, 2015.

NETO, C. L. de B. et al. Teleodontologia. In: SEDIS/UFRN (Ed.). A Telessaúde no Brasil e a Inovação Tecnológica na Atenção Primária. 2. ed. Natal: [s.n.], 2018. p. 105-138.

NILSON, L. G. et al. Telessaúde: Da Implantação Ao Entendimento Como Tecnologia Social. Revista Brasileira de Tecnologias Sociais, v. 5, n. 1, p. 33, 2018. 
SCHMITZ, C. A. A.; HARZHEIM, E. Oferta e utilização de teleconsultorias para Atenção Primária à Saúde no Programa Telessaúde Brasil Redes. Revista Brasileira de Medicina de Família e Comunidade, v. 12, n. 39, p. 1-11, 2017.

SILVA, E. A. A Telessaúde e seus impactos na formação continuada dos profissionais de saúde em Rede. EmRede - Revista de

Educação a Distância, v. 4, n. 1, 2017.

GEREMIAS, M. A.; JACOBSEN, A. L.; PEREIRA, J. Superação dos Desafios na Integração dos Sistemas de Informação em Saúde na Secretaria Municipal de Saúde de Florianópolis. In: PEREIRA, M. F. et al. (org.). Contribuições para a Gestão do SUS. Florianópolis: Fundação Boiteux, 2013. 231p. (Gestão da Saúde Pública; v. 8).

PREFEITURA MUNICIPAL DE NATAL. 2018 - Disponível em: https://www.natal.rn.gov.br/noticia/ntc-27893.html. Acessado em: 22 set. 2018.

SEGUNDA OPINIÃO FORMATIVA (SOF). Núcleo de Telessaúde do Rio Grande do Sul. Quantos pacientes doentes o exame de colonoscopia pode detectar? qual sua sensibilidade? Atenção Primária à Saúde, São Paulo, mar. 2013. Disponível em: http://aps. bvs.br/aps/quantos-pacientes-doentes-o-exame-de-colonoscopiapode-detectar-qual-sua-sensibilidade/. Acesso em: 4 ago. 2018. 